\title{
Modelo matemático de los factores que influyen en el rendimiento académico de estudiantes universitarios ingresantes
}

\author{
Mathematical model of factors that influence the academic performance of \\ students entering
}

${ }^{1}$ Humberto Benito Vargas Pichon

${ }^{2}$ Wilsón Chanini Choquecota
ORCID: 0000-0003-4892-5275

ORCID: 0000-0001-9792-3770

\section{RESUMEN}

El objetivo de esta investigación fue comprobar en qué medida un modelo matemático explica qué factores influyen significativamente en el rendimiento académico de los estudiantes ingresantes a la Universidad Nacional Jorge Basadre Grohmann, años académico 2018 y 2019. El tipo del estudio fue no-experimental. Para el año académico 2018, se consideró una población de 1754 estudiantes ingresantes y una muestra de 1526 estudiantes. Por otro lado, para el año académico 2019, se consideró una población de 1756 estudiantes ingresantes y una muestra de 1455 estudiantes. Se tuvo como resultado que Existe una relación directa y significativa entre el puntaje de examen de ingreso y el rendimiento académico de los estudiantes ingresantes de la Universidad Nacional Jorge Basadre Grohmann, detectado con la correlación de Pearson $\mathrm{r}=0,380 ; \mathrm{r}=0,306$ y una significancia al $99 \%$ para los años 2018 y 2019 respectivamente. Así, se obtuvo los modelos matemáticos y=6,304+0,015x, con $\mathrm{R}$ cuadrado =0,144 para el año 2018; e y=9,832+0,0009x, con $\mathrm{R}$ cuadrado =0,094 para el año 2019.

Palabras claves: Puntaje de examen de ingreso, rendimiento académico.

\begin{abstract}
The objective of this research was to verify to what extent a mathematical model explains which factors significantly influence the academic performance of students entering the Universidad Nacional Jorge Basadre Grohmann, academic years 2018 and 2019. The type of study was non-experimental. For the 2018 academic year, a population of 1754 incoming students and a sample of 1526 students were considered. On the other hand, for the 2019 academic year, a population of 1756 incoming students and a sample of 1455 students were considered. The result was that there is a direct and significant relationship between the entrance exam score and the academic performance of the incoming students of the Jorge Basadre Grohmann National University, detected with the Pearson correlation $r=0,380, r$ $=0,306$ and a significance of $99 \%$ for the years 2018 and 2019 respectively. Thus, the mathematical models $y=6,304+0,015 x$, with $R$ squared $=0,144$ for the year 2018 and $y=9,832+0.0009 x$, with $R$ squared $=0,094$ for the year 2019 .
\end{abstract}

Keywords: Entrance exam score, academic performance.

\footnotetext{
${ }^{1}$ Universidad Nacional Jorge Basadre Grohmann. Tacna, Perú. E-mail: humberto.vargas@unjbg.edu.pe

${ }^{2}$ Universidad Nacional Jorge Basadre Grohmann. Tacna, Perú. E-mail: wchaninic@unjbg.edu.pe
} 


\section{INTRODUCCIÓN}

El acceso a la educación universitaria selectiva, en países como Chile, ordena a los postulantes de acuerdo a un puntaje que pondera los factores de trayectoria escolar y prueba de selección universitaria (PSU). En 2013, la inclusión del ranking de notas ocasionó una reestructuración en la ponderación de los factores de selección, redefiniendo las posiciones institucionales al respecto.

Con bases de datos oficiales y procedimientos estadísticos ad hoc, se describe el comportamiento de los 1423 programas de pre-grado ofertados por las 33 universidades integrantes del Sistema Único de Admisión en 2014. Se observa que el componente PSU pierde hegemonía comparado con la trayectoria escolar como factor de ponderación, resignificándose especialmente el ranking. Esta es una tendencia generalizada, pero de comportamiento no homogéneo (Rodríguez \& Padilla, 2016).

Si bien el rendimiento académico del estudiantado que ingresa a las universidades chilenas ha sido objeto de múltiples estudios, pocas investigaciones han analizado la relación que tienen los instrumentos de selección de ingreso y el rendimiento académico posterior. En una muestra de 440 estudiantes de Ingeniería Comercial de la Universidad Austral de Chile, pertenecientes a cinco promociones de primer año, se estudiaron los instrumentos de admisión a la carrera, y se determinó cuáles explican mejor el rendimiento académico. Para ello se utilizaron los resultados de las PSU y las notas de enseñanza media (NEM) obtenidos por el estudiantado, en relación con el promedio semestral ponderado (PSP) del primer año universitario. Se determinó que el promedio de NEM explica mejor el rendimiento académico estudiantil, al presentar la mejor correlación con el rendimiento académico del primer semestre y de manera más fuerte en el segundo (Vergara \& Peredo, 2017).

A nivel de Perú tenemos la investigación Relación entre las modalidades de ingreso y el rendimiento académico de los estudiantes de estomatología de las cohortes 2008-2009 en una Universidad Privada. Cuyo objetivo fue determinar la relación entre las modalidades de ingreso y el rendimiento académico universitario de los estudiantes de estomatología de las cohortes 2008-2009 de la Universidad Peruana Cayetano Heredia (UPCH). El diseño fue de corte descriptivo, observacional, longitudinal y retrospectivo, se realizó en la población total de estudiantes de la Facultad de Estomatología Roberto Beltrán de la UPCH que ingresaron en los años 2008 y 2009 que cursaron el primer y segundo año académico. En el 2008 ingresaron 118 y en el 2009 fueron 110. Se tuvo como resultados que respecto a las modalidades de ingreso se observa que el mayor número de estudiantes ingresaron por Factor Excelencia $(\mathrm{N}=135,59 \%)$, seguido de Examen de admisión ( $\mathrm{N}=52,22 \%)$ y Centro Preuniversitario-CEPREU (N=38, 16\%), respecto al rendimiento académico universitario según género se encontró que el mayor número de estudiantes fueron de sexo femenino $(\mathrm{N}=159,70 \%)$ seguido del sexo masculino $(\mathrm{N}=69,30 \%)$, no se encontró diferencias estadísticamente significativas respecto al rendimiento académico universitario según sexo. Las conclusiones fueron que respecto al estudio de la relación del rendimiento académico universitario con las modalidades de ingreso se encontró que los estudiantes obtuvieron promedios similares en el rendimiento académico universitario al margen de la modalidad de ingreso, en términos de promedio ponderado anual, la relación no fue estadísticamente significativa $(\mathrm{P}>0,05)$. Las modalidades de admisión parecen no ser un buen predictor del rendimiento académico; salvo el rendimiento escolar previo, una vez que el estudiante ingresa a la 
universidad se disipan algunas variables que los caracterizan durante la etapa escolar. (Flores, 2012, p. 210).

Por otro lado, a nivel local tenemos la Investigación Rendimiento académico de ingreso y su relación con el rendimiento académico en el primer año de educación universitaria de la UNJBG en año 2006. El objetivo de la investigación fue determinar el grado de relación que existe entre el rendimiento académico de secundaria con el rendimiento académico alcanzado por el estudiante en el primer año de educación universitaria en la Universidad Nacional Jorge Basadre Grohmann de Tacna, en el año 2006. El método fue empírico, basado en análisis documental de rendimiento de ingreso y rendimiento alcanzado por los estudiantes según actas finales de evaluación. Las conclusiones a las que se arribó al finalizar esta investigación fueron: 1) El rendimiento académico de los estudiantes de secundaria al ingresar a la universidad se concentra en la categoría de regular a bueno, pues no hay desaprobados, con un 56,3\% (regular) y un 43,1\% (bueno). 2) El rendimiento académico de los estudiantes en el primer año de estudios universitarios se concentra en la categoría de regular con un 49,7\% (regular) y un $17,9 \%$ (bueno). 3 ) El 32,4 \% de estudiantes disminuyen su rendimiento académico en el primer año de universidad y pasan de la condición de aprobados al ingresar a la universidad a la condición de desaprobados (Chávez \& Mendoza, 2006, p. 47).

También, se tiene la investigación Relación entre el examen de admisión y el académico, 2001-2005. Donde se presentan los resultados de un estudio transversal retrospectivo que tuvo por objetivo establecer la relación entre el puntaje del examen de ingreso y el rendimiento académico de los ingresantes del 2001 a la Facultad de Ciencias de la Educación de la UNJBG. Se determinó que existe relación directa $(\mathrm{p}<0,05)$ con una baja correlación $(\mathrm{r}=0,34)$, esto, con escaso significado estadístico (Benites \& Ticona, 2006, $\mathrm{p}$. $15)$.

\section{Sobre exámenes de admisión en las universidades}

La educación superior se ha visto enfrentada a retos sin precedente desde inicios del siglo XXI, bajo el impacto de la globalización y el crecimiento económico, además de la revolución de la información y la comunicación. Estos cambios trascendentales han obligado a las instituciones a mejorar la calidad de la educación que ofrecen, por lo que se han replanteado los mecanismos de selección de los aspirantes para que, a través de esta selección, se garantice la formación de egresados de la más alta calidad académica que sean competitivos para ocupar los reducidos espacios que ofrece el mercado laboral (Cortés \& Palomar, 2008).

Para asegurar la calidad de la educación superior, se debe partir de la selección de los estudiantes considerando los factores que influyen sobre el rendimiento académico y que repercuten en la deserción estudiantil sobre todo durante la transición del colegio a la universidad. La universidad solo debe admitir como estudiantes a aquellas personas que tengan un legítimo y autentico interés por el conocimiento como factor necesario para el avance social (Flores, 2012).

El objetivo de los procesos de selección es obtener información de un conjunto de examinados sobre algún dominio específico, como habilidades o conocimientos, mediante la asignación de puntajes. Un ejemplo de este tipo de procesos es el ingreso a la educación superior, en el que se utiliza una serie de instrumentos que permiten tomar 
decisiones sobre los participantes y las carreras o universidades a las que postulan. Así, el diseño apropiado de los mecanismos de selección es clave, ya que si estos no son adecuados pueden generar un significativo perjuicio para los postulantes, ya que tarde o temprano pueden fracasar en sus estudios (Larroucau et al, 2015).

\section{Sobre rendimiento académico en las universidades}

Para el desarrollo de un país es de gran importancia la formación y calificación de su capital humano. En ese sentido, las universidades juegan un rol muy importante al ser las responsables de la formación de profesionales, junto a las instituciones de educación superior técnico-profesional e institutos pedagógicos. Cada vez más se incrementa el interés de las universidades y de los gobiernos por el rendimiento académico y, especialmente, por el abandono de los estudios, como se evidencia en las políticas de estado en relación a evaluar la calidad de las universidades. Tal es el caso del Perú, que en 2014 promulgó una nueva Ley Universitaria, crea la Superintendencia Nacional de Educación Superior Universitaria (SUNEDU) y se establece el licenciamiento obligatorio y renovable de las universidades, en lugar de la autorización de funcionamiento del anterior marco legal. Posteriormente, se aprobó la Política de Aseguramiento de la Calidad de la Educación Superior Universitaria la misma que establece el proceso de licenciamiento obligatorio como uno de los cuatro pilares del Sistema de Aseguramiento de la Calidad (https://www.sunedu.gob.pe). Las tendencias de la educación superior han obligado a estas a diseñar estrategias, que permitan incrementar la calidad de la formación y a evaluar constantemente su quehacer, con la intención de lograr un mejor rendimiento académico en los estudiantes y su permanencia hasta lograr terminar sus estudios profesionales en un periodo adecuado. El rendimiento académico alude al promedio de calificaciones obtenido por el alumno, en las asignaturas, en las cuales ha presentado examen, independientemente del tipo de examen. Su indicador es la suma total de calificaciones obtenidas, divididas entre el número de calificaciones. La autora continúa afirmando que el rendimiento escolar o académico es el nivel de conocimientos demostrado en un área o materia, comparado con la norma (edad, nivel académico) y las calificaciones suelen reflejar las preferencias, la dedicación y la capacidad mayor o menor de los alumnos en distintas materias (Treviño-Lozano, 2003).

\section{MATERIAL Y MÉTODOS}

\section{Enfoque metodológico}

La investigación centró el desarrollo de su metodología dentro del paradigma positivista, bajo un enfoque cuantitativo, de tipo aplicada, correlacional pues demuestra la relación que existe entre ambas variables de estudio, y de diseño no experimental, descriptivo, correlacional, predictivo y retrospectivo.

\section{Variables de estudio}

Puntaje de examen de ingreso y rendimiento académico.

Para el año académico 2018, se consideró una población de 1754 estudiantes ingresantes a la UNJBG y una muestra de 1526 estudiantes. Por otro lado, para el año académico 2019, se consideró una población de 1756 estudiantes ingresantes a la UNJBG y una muestra de 1455. Las muestras fueron seleccionadas por conveniencia (se excluyó a 228 estudiantes del año 2018 y 230 estudiantes del 2019). 


\section{Instrumentos de recolección de datos}

Para la variable Puntaje de examen de ingreso se usó la técnica del análisis documental y como instrumento los registros de notas de la Oficina de Admisión y/o Centro Pre Universitario (CEPU) que ingresaron en los años 2018 y 2019. Para la variable Rendimiento académico se utilizó la técnica del análisis documental y como instrumento el ranking académico de los estudiantes ingresantes a la Universidad Nacional Jorge Basadre Grohmann, años académicos 2018 y 2019 (DASA-UNJBG).

\section{Validación de instrumentos}

Según la información recabada de la Dirección Académica de Actividades y Servicios Académicos (DASA) y el Ranking Académico UNJBG 2018 y 2019.

\section{Validación de las escalas de valores utilizadas}

Según criterio de la UNJBG, para la variable puntaje de examen de admisión una escala de 00 a 600 y para la variable rendimiento académico una escala de 00 a 20.

\section{Análisis estadístico y presentación de los datos}

Estadística descriptiva: Mínimo, máximo, media y desviación estándar.

\section{Estadística inferencial: Análisis inferencial cuantitativo}

Se hizo usando la correlación de Pearson y regresión lineal, con un nivel de significancia de $\alpha=0,05$ y con una confiabilidad del $95 \%$.

\section{RESULTADOS}

Usando el software estadístico SPSS versión 20 se obtuvo los siguientes resultados:

Tabla 1

Porcentaje de estudiantes ingresantes del año académico 2018, según sexo

\begin{tabular}{llcccc}
\hline & Frecuencia & $\begin{array}{c}\text { Porcentaj } \\
\text { e }\end{array}$ & Porcentaje válido & $\begin{array}{c}\text { Porcentaje } \\
\text { acumulado }\end{array}$ \\
\hline \multirow{2}{*}{ Válidos } & Femenino & 712 & 46,7 & 46,7 & 46,7 \\
\cline { 2 - 6 } & Masculino & 814 & 53,3 & 53,3 & 100,0 \\
\cline { 2 - 5 } & Total & 1526 & 100,0 & 100,0 & \\
\hline
\end{tabular}

Fuente: Matriz de sistematización de datos 2018, marzo 2020

De los estudiantes ingresantes a la UNJBG el año académico 2018, el 53,3\% correspondieron al sexo masculino y el $46,7 \%$ al sexo femenino. 
Modelo matemático de los factores que influyen en el rendimiento académico de estudiantes universitarios

Tabla 2

Estudiantes ingresantes del año académico 2019, según sexo

\begin{tabular}{llcccc}
\hline & & Frecuencia & Porcentaje & Porcentaje válido & Porcentaje acumulado \\
\hline \multirow{2}{*}{ Válidos } & Femenino & 685 & 47,1 & 47,1 & 47,1 \\
\cline { 2 - 6 } & Masculino & 770 & 52,9 & 52,9 & 100,0 \\
\cline { 2 - 6 } & Total & 1455 & 100,0 & 100,0 & \\
\hline
\end{tabular}

Fuente: Matriz de sistematización de datos 2019, marzo 2020

De los estudiantes ingresantes a la UNJBG el año académico 2019, el 52,92\% correspondieron al sexo masculino y el $47,08 \%$ al sexo femenino.

Tabla 3

Porcentaje de estudiantes ingresantes del año académico 2018, según tipo de institución educativa de procedencia

\begin{tabular}{llcccc}
\hline & Frecuencia & Porcentaje & $\begin{array}{c}\text { Porcentaje } \\
\text { válido }\end{array}$ & $\begin{array}{c}\text { Porcentaje } \\
\text { acumulado }\end{array}$ \\
\hline \multirow{3}{*}{ Válidos } & Estatal & 1071 & 70,2 & 70,2 & 70,2 \\
\cline { 2 - 6 } & No especificado & 17 & 1,1 & 1,1 & 71,3 \\
\cline { 2 - 6 } & Privado & 438 & 28,7 & 28,7 & 100,0 \\
\cline { 2 - 6 } & Total & 1526 & 100,0 & 100,0 & \\
\hline
\end{tabular}

Fuente: Matriz de sistematización de datos 2018, marzo 2020

De los estudiantes ingresantes a la UNJBG el año académico 2018, el 70,18\% procedieron de una institución educativa estatal; el 28,70\% de una privada y el 1,11\% no mencionaron el tipo de institución educativa de procedencia.

\section{Tabla 4}

Estudiantes ingresantes del año académico 2019, según tipo de Institución Educativa de procedencia

\begin{tabular}{llcccc}
\hline & Frecuencia & Porcentaje & $\begin{array}{c}\text { Porcentaje } \\
\text { válido }\end{array}$ & $\begin{array}{c}\text { Porcentaje } \\
\text { acumulado }\end{array}$ \\
\hline \multirow{3}{*}{ Válidos } & Estatal & 1047 & 72,0 & 72,0 & 72,0 \\
\cline { 2 - 6 } & No especificado & 7 &, 5 &, 5 & 72,4 \\
\cline { 2 - 6 } & Privado & 401 & 27,6 & 27,6 & 100,0 \\
\cline { 2 - 6 } & Total & 1455 & 100,0 & 100,0 & \\
\hline
\end{tabular}

Fuente: Matriz de sistematización de datos 2019, marzo 2020

De los estudiantes ingresantes a la UNJBG el año académico 2019, el 71,96\% procedieron de una Institución Educativa Estatal; el 27,56\% de una Privada y el 0,48\% no mencionaron el tipo de Institución Educativa de procedencia. 
Modelo matemático de los factores que influyen en el rendimiento académico de estudiantes universitarios

Tabla 5

Porcentaje de estudiantes ingresantes del año académico 2018, según Modalidad de ingreso

\begin{tabular}{llcccc}
\hline & Frecuencia & Porcentaje & $\begin{array}{c}\text { Porcentaje } \\
\text { válido }\end{array}$ & $\begin{array}{c}\text { Porcentaje } \\
\text { acumulado }\end{array}$ \\
\hline Válidos & Admisión & 1029 & 67,4 & 67,4 & 67,4 \\
\cline { 2 - 6 } & CEPU & 497 & 32,6 & 32,6 & 100,0 \\
\cline { 2 - 6 } & Total & 1526 & 100,0 & 100,0 & \\
\hline
\end{tabular}

Fuente: Matriz de sistematización de datos 2018, marzo 2020

De los estudiantes ingresantes a la UNJBG el año académico 2018, el 67,43\% ingresaron bajo la modalidad de examen de ADMISIÓN y el 32,57\% bajo la modalidad de examen de CEPU.

Tabla 6

Porcentaje de estudiantes ingresantes del año académico 2019, según Modalidad de ingreso

\begin{tabular}{lcccc}
\hline & Frecuencia & Porcentaje & $\begin{array}{c}\text { Porcentaje } \\
\text { válido }\end{array}$ & $\begin{array}{c}\text { Porcentaje } \\
\text { acumulado }\end{array}$ \\
\hline CEPU - I (Otoño) & 153 & 10,5 & 10,5 & 10,5 \\
\hline CEPU - II (Invierno) & 174 & 12,0 & 12,0 & 22,5 \\
\hline CEPU - III (Verano) & 169 & 11,6 & 11,6 & 34,1 \\
\hline FASE I & 267 & 18,4 & 18,4 & 52,4 \\
\hline FASE II & 558 & 38,4 & 38,4 & 90,8 \\
\hline $\begin{array}{l}\text { Ley 27050 Persona con } \\
\text { Discapacidad }\end{array}$ & 4 &, 3 &, 3 & 91,1 \\
\hline $\begin{array}{l}\text { Ley de Promoción y Desarrollo } \\
\text { del Deporte }\end{array}$ & 16 & 1,1 & 1,1 & 92,2 \\
\hline $\begin{array}{l}\text { Primeros Puestos Colegios y } \\
\text { COAR }\end{array}$ & 89 & 6,1 & 6,1 & 98,3 \\
\hline $\begin{array}{l}\text { Titulados/Graduados de Univ. } \\
\text { o de rango de Univ. }\end{array}$ & 16 & 1,1 & 1,1 & 99,4 \\
\hline Traslado Externo & 1 &, 1 &, 1 & 99,5 \\
\hline Traslado Interno & 8 &, 5 &, 5 & 100,0 \\
\hline Total & 1455 & 100,0 & 100,0 & \\
\hline
\end{tabular}

Fuente: Matriz de sistematización de datos 2019, marzo 2020

De los estudiantes ingresantes a la UNJBG el año académico 2019, el 65,90\% ingresaron bajo la modalidad de examen de ADMISIÓN (Fase I, fase II y Otros) y el 34,10\% bajo la modalidad de examen de CEPU (otoño, invierno y verano).

Tabla 7

Estadísticos descriptivos de los factores Edad, Rendimiento académico 2018 y Puntaje de Examen de Ingreso

\begin{tabular}{lccccc}
\hline & N & Mínimo & Máximo & Media & Desv. típ. \\
\hline Edad & 1526 & 16 & 44 & 19,54 & 2,661 \\
\hline Rendimiento académico 2018 & 1526 &, 09 & 17,59 & 10,7000 & 3,68073 \\
\hline Puntaje de examen de ingreso & 1526 & 80,00 & 580,50 & 296,2641 & 94,18748 \\
\hline N válido (según lista) & 1526 & & & & \\
\hline
\end{tabular}

Fuente: Matriz de sistematización de datos 2018, marzo 2020 
El promedio de edad de los estudiantes ingresantes a la Universidad Nacional Jorge Basadre Grohmann, año académico 2018, fue de 19,54 años. Teniendo en cuenta como edad mínima de 16 años; edad máxima de 44 años y teóricamente el promedio de los alejamientos de los datos respecto a la media (desviación estándar) fue de 2,66. Por otro lado, el promedio del rendimiento académico 2018 fue de 10,70. Teniendo en cuenta como mínimo el rendimiento académico de 0,09, el máximo de 17,59 y teóricamente el promedio de los alejamientos de los datos respecto a la media (desviación estándar) fue de 3,68. Finamente, el promedio del puntaje de examen de ingreso fue de 296,26. Teniendo en cuenta como puntaje mínimo de ingreso de 80,0; el máximo de 580,5 y teóricamente el promedio de los alejamientos de los datos respecto a la media (desviación estándar) fue de 94,19.

\section{Tabla 8}

Estadísticos descriptivos de los factores edad, rendimiento académico 2019 y puntaje de examen de ingreso

\begin{tabular}{|c|c|c|c|c|c|}
\hline & $\mathbf{N}$ & Mínimo & $\begin{array}{c}\text { Máxim } \\
\text { o }\end{array}$ & Media & Desv. típ. \\
\hline Edad & 1455 & 16 & 65 & 19,25 & 3,547 \\
\hline $\begin{array}{ll}\text { Rendimiento } & \text { Académico } \\
\text { UNJBG } 2019 & \end{array}$ & 1455 &, 00 & 17,57 & 12,2134 & 2,76425 \\
\hline Puntaje de examen & 1455 & 27,63 & 575,00 & 277,9343 & 98,86943 \\
\hline $\mathbf{N}$ válido (según lista) & 1455 & & & & \\
\hline
\end{tabular}

Fuente: Matriz de sistematización de datos 2019, marzo 2020

El promedio de edad de los estudiantes ingresantes a la Universidad Nacional Jorge Basadre Grohmann, año académico 2019, fue de 19,25 años. Teniendo en cuenta como edad mínima de 16 años, edad máxima de 65 años; teóricamente, el promedio de los alejamientos de los datos respecto a la media (desviación estándar) fue de 3,55. Por otro lado, el promedio del rendimiento académico 2019 fue de 12,21. Teniendo en cuenta como mínimo el rendimiento académico de 0,00, el máximo de 17,57 y teóricamente el promedio de los alejamientos de los datos respecto a la media (desviación estándar) fue de 2,76. Finamente, el promedio del puntaje de examen de ingreso fue de 277,93. Teniendo en cuenta como puntaje mínimo de ingreso de 27,63; el máximo de 575,00 y teóricamente el promedio de los alejamientos de los datos respecto a la media (desviación estándar) fue de 98,87. 
Modelo matemático de los factores que influyen en el rendimiento académico de estudiantes universitarios

Tabla 9

Matriz de correlaciones de los factores Edad, Rendimiento académico 2018 y Puntaje de Examen de Ingreso

\begin{tabular}{|c|c|c|c|c|}
\hline & & Edad & $\begin{array}{c}\text { Rendimient } \\
\text { o académico } \\
2018\end{array}$ & $\begin{array}{c}\text { Puntaje de examen } \\
\text { de ingreso }\end{array}$ \\
\hline \multirow{3}{*}{ Edad } & Correlación de Pearson & 1 &,- 003 &,- 016 \\
\hline & Sig. (bilateral) & & ,914 &, 534 \\
\hline & $\mathrm{N}$ & 1526 & 1526 & 1526 \\
\hline \multirow{3}{*}{$\begin{array}{l}\text { Rendimiento } \\
\text { académico } 2018\end{array}$} & Correlación de Pearson &,- 003 & 1 &, $380^{* *}$ \\
\hline & Sig. (bilateral) & 914 & &, 000 \\
\hline & $\mathrm{N}$ & 1526 & 1526 & 1526 \\
\hline \multirow{3}{*}{$\begin{array}{l}\text { Puntaje de examen } \\
\text { de ingreso }\end{array}$} & Correlación de Pearson &,- 016 & ,380** & 1 \\
\hline & Sig. (bilateral) &, 534 &, 000 & \\
\hline & $\mathrm{N}$ & 1526 & 1526 & 1526 \\
\hline
\end{tabular}

**. La correlación es significativa al nivel 0,01 (bilateral).

Fuente: Matriz de sistematización de datos 2018, marzo 2020

Vemos que existe una relación directa y significativa entre los factores rendimiento académico 2018 y puntaje de examen de ingreso. Verificado con una correlación de Pearson $r=0,380$ con un nivel de significancia del 99\%. Por otro lado, el factor edad no tiene relación con los factores antes mencionados.

Tabla 10

Matriz de correlaciones de los factores Edad, Rendimiento académico 2019 y Puntaje de Examen de Ingreso

\begin{tabular}{|c|c|c|c|c|}
\hline & & Edad & $\begin{array}{c}\text { Rendimiento } \\
\text { Académico } \\
2019\end{array}$ & Puntaje de examen \\
\hline \multirow{3}{*}{ Edad } & Correlación de Pearson & 1 &,- 004 & ,033 \\
\hline & Sig. (bilateral) & & ,866 & ,208 \\
\hline & $\mathrm{N}$ & 1455 & 1455 & 1455 \\
\hline \multirow{3}{*}{$\begin{array}{l}\text { Rendimiento } \\
\text { Académico } 2019\end{array}$} & Correlación de Pearson &,- 004 & 1 &, $306^{* *}$ \\
\hline & Sig. (bilateral) &, 866 & &, 000 \\
\hline & $\mathrm{N}$ & 1455 & 1455 & 1455 \\
\hline \multirow{3}{*}{ Puntaje de examen } & Correlación de Pearson &, 033 &, $306^{* *}$ & 1 \\
\hline & Sig. (bilateral) & ,208 &, 000 & \\
\hline & $\mathrm{N}$ & 1455 & 1455 & 1455 \\
\hline
\end{tabular}

**. La correlación es significativa al nivel 0,01 (bilateral).

Fuente: Matriz de sistematización de datos 2019, marzo 2020

Vemos que existe una relación directa y significativa entre los factores rendimiento académico 2019 y puntaje de examen de ingreso. Verificado con una correlación de Pearson $\mathrm{r}=0,306$ con un nivel de significancia del $99 \%$. Por otro lado, el factor edad no tiene relación con los factores antes mencionados. 
Modelo matemático de los factores que influyen en el rendimiento académico de estudiantes universitarios

\section{Tabla 11}

Resumen del modelo y estimaciones de los parámetros para el año 2018 Variable dependiente: Rendimiento académico 2018

\begin{tabular}{|c|c|c|c|c|c|c|c|}
\hline \multirow[t]{2}{*}{ Ecuación } & \multicolumn{5}{|c|}{ Resumen del modelo } & \multicolumn{2}{|c|}{$\begin{array}{c}\text { Estimaciones de los } \\
\text { parámetros }\end{array}$} \\
\hline & R cuadrado & $\mathrm{F}$ & gl1 & $\mathrm{g} 12$ & Sig. & $\begin{array}{c}\text { Consta } \\
\text { nte }\end{array}$ & b1 \\
\hline Lineal &, 144 & $\begin{array}{c}256,77 \\
4\end{array}$ & 1 & 1524 & ,000 & 6,304 & ,015 \\
\hline
\end{tabular}

La variable independiente es Puntaje de examen de ingreso.

Fuente: Matriz de sistematización de datos 2018, marzo 2020

\section{Figura 1}

Dispersión de la variable puntaje de examen de ingreso vs rendimiento académico 2018

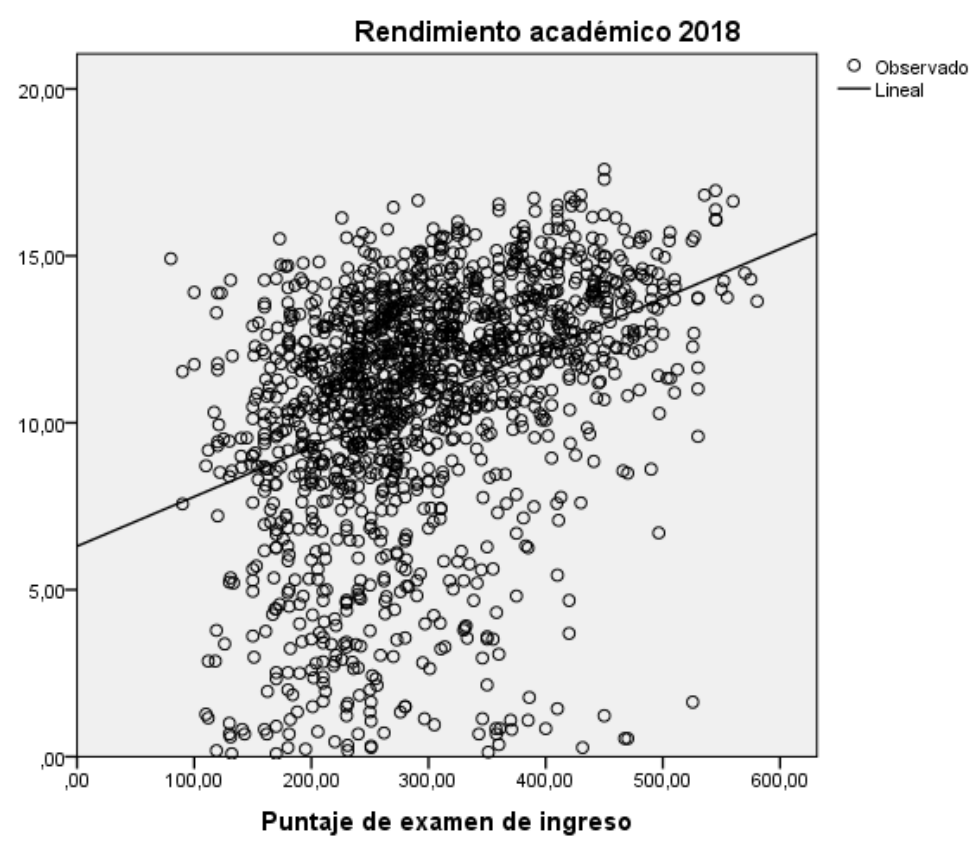

Fuente: Matriz de sistematización de datos 2018, marzo 2020

Se observa que ambas variables presentan una relación lineal positiva; con un coeficiente de determinación de $\boldsymbol{R}$ cuadrado $=\mathbf{0 , 1 4 4}$. Es decir, las variables están relacionadas en un $14,4 \%$. Así, se obtiene el modelo lineal:

$$
y=6,304+0,015 x
$$


Modelo matemático de los factores que influyen en el rendimiento académico de estudiantes universitarios

Donde:
$\mathbf{y}=$ Rendimiento Académico 2018
$0,09 \leq \mathrm{y} \leq 17,59$
$\mathbf{x}=$ Puntaje de examen de ingreso
$80,00 \leq x \leq 580,50$

\section{Aplicación del Modelo Matemático}

¿Qué puntaje de rendimiento académico 2018 esperaría un estudiante con puntaje de examen de ingreso de 500?

Respuesta: $\quad \mathrm{y}=6,304+0,015(500)=13,804$

Tabla 12

Resumen del modelo y estimaciones de los parámetros para el año 2019

Variable dependiente: rendimiento académico UNJBG 2019

\begin{tabular}{lccccccc} 
Ecuación & \multicolumn{4}{c}{ Resumen del modelo } & \multicolumn{3}{c}{$\begin{array}{c}\text { Estimaciones de los } \\
\text { parámetros }\end{array}$} \\
\cline { 2 - 9 } & $\begin{array}{c}\mathrm{R} \\
\text { cuadrado }\end{array}$ & $\mathrm{F}$ & $\mathrm{gl1}$ & $\mathrm{gl} 2$ & Sig. & $\begin{array}{c}\text { Consta } \\
\text { nte }\end{array}$ & b1 \\
\hline Lineal &, 094 & $\begin{array}{c}150,61 \\
0\end{array}$ & 1 & 1453 &, 000 & 9,832 &, 009 \\
\hline
\end{tabular}

La variable independiente es Puntaje de examen.

Fuente: Matriz de sistematización de datos 2019, marzo 2020

Figura 2

Dispersión de la variable puntaje de examen de ingreso vs rendimiento académico 2019

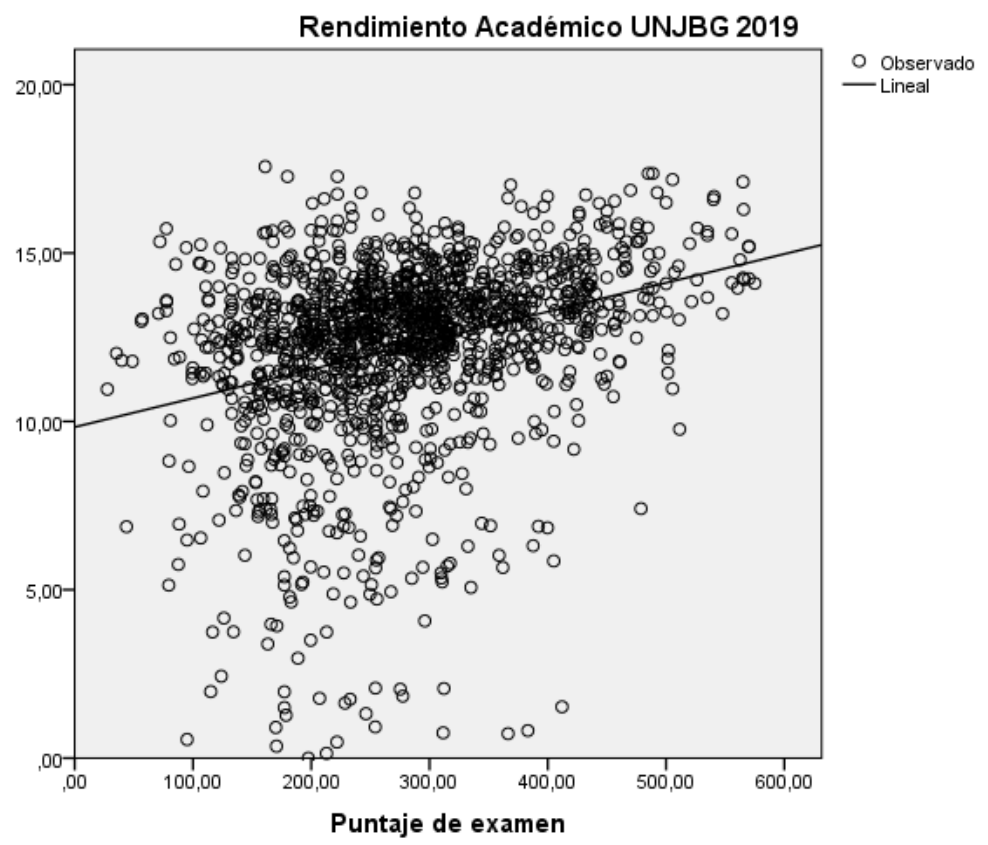

Fuente: Matriz de sistematización de datos 2019, marzo 2020 
Se observa que ambas variables presentan una relación lineal positiva; con un coeficiente de determinación de $\boldsymbol{R}$ cuadrado $=\mathbf{0 , 0 9 4}$. Es decir, las variables están relacionadas en un $9,4 \%$. Así, se obtiene el modelo lineal:

$y=9,832+0,009 x$

Donde:

$$
\begin{array}{lc}
\mathbf{y}=\text { Rendimiento Académico } 2019 & 0,00 \leq y \leq 17,57 \\
\mathbf{x}=\text { Puntaje de examen de ingreso } & 27,63 \leq x \leq 575,00
\end{array}
$$

\section{Aplicación del Modelo Matemático}

¿Qué puntaje de rendimiento académico 2019 esperaría un estudiante con puntaje de examen de ingreso de 500?

\section{Respuesta:}

$$
\mathrm{y}=9,832+0,009(500)=14,332
$$

\section{Verificación de las hipótesis}

\section{Hipótesis específicas}

a) Según las Tablas 9 y 10, NO existe una relación directa y significativa entre el factor edad y el rendimiento académico de los estudiantes ingresantes de la Universidad Nacional Jorge Basadre Grohmann, años académicos 2018 y 2019. Por tanto, la edad $\mathrm{NO}$ es un factor que influye significativamente en el rendimiento académico de los estudiantes ingresantes de la UNJBG, años académicos 2018 y 2019.

b) Según las Tablas 9 y 10, existe una relación directa y significativa entre el factor puntaje examen de ingreso y el rendimiento académico de los estudiantes ingresantes de la Universidad Nacional Jorge Basadre Grohmann, años académico 2018 y 2019. Detectado con una correlación de Pearson con un $r=0,380$ para el año 2018, r=0,306 para el año 2019 y una significancia al $99 \%$. Así, el puntaje de examen de ingreso es un factor que influye significativamente en el rendimiento académico de los estudiantes ingresantes de la UNJBG, años académicos 2018 y 2019.

\section{Hipótesis general}

Según la Tabla 11 y la Figura 1, el modelo matemático obtenido para el año académico 2018 es:

$$
\mathrm{y}=6,304+0,015 \mathrm{x}
$$

Donde:

$\mathbf{y}=$ Rendimiento académico $2018 \quad 0,09 \leq \mathrm{y} \leq 17,59$

$\mathbf{x}=$ Puntaje de examen de ingreso $\quad 80,00 \leq x \leq 580,50$

Detectado con un coeficiente de determinación de $\mathbf{R}$ cuadrado $=0,144$ y una significancia al $99 \%$.

Similarmente, según la Tabla 12 y Gráfica 2, el modelo matemático obtenido para el año académico 2019 es:

$$
\mathrm{y}=9,832+0,009 x
$$


Donde:

$\mathbf{y}=$ Rendimiento Académico 2019

$0,00 \leq \mathrm{y} \leq 17,57$

$\mathbf{x}=$ Puntaje de examen de ingreso

$27,63 \leq x \leq 575,00$

Detectado con un coeficiente de determinación de $\mathbf{R}$ cuadrado $=\mathbf{0 , 0 9 4}$ y una significancia al $99 \%$.

\section{DISCUSIÓN}

A partir de los resultados hallados de la hipótesis general, el puntaje de examen de ingreso es un factor que influye significativamente en el rendimiento académico de los estudiantes ingresantes de la Universidad Nacional Jorge Basadre Grohmann, años académicos 2018 y 2019.

Estos resultados guardan relación con lo que sostienen Vergara \& Peredo (2017), quienes señalan que existe relación entre los instrumentos de selección de ingreso y el rendimiento académico posterior. Ello es acorde con lo que en este estudio se halla.

\section{CONCLUSIONES}

En estudiantes ingresantes de la Universidad Nacional Jorge Basadre Grohmann, años académicos 2018 y 2019, la edad no es un factor que influye significativamente en el rendimiento académico.

En estudiantes ingresantes de la Universidad Nacional Jorge Basadre Grohmann, años académicos 2018 y 2019, el Puntaje de examen de ingreso es un factor que influye significativamente en el rendimiento académico.

En estudiantes ingresantes de la Universidad Nacional Jorge Basadre Grohmann, años académicos 2018 y 2019; los modelos matemáticos respectivos son:

$\mathrm{y}=6,304+0,015 \mathrm{x}$

$\mathrm{y}=9,832+0,009 \mathrm{x}$

Donde:

$$
\begin{aligned}
& \mathbf{y}=\text { Rendimiento Académico } \\
& \mathbf{x}=\text { Puntaje de examen de ingreso }
\end{aligned}
$$

\section{RECOMENDACIONES}

Ampliar y profundizar la investigación, tomando en cuenta los factores: trayectoria escolar, tipo de institución educativa de procedencia y sexo.

Reformular el examen de ingreso a fin de obtener mejores resultados, considerando el ranking académico, entrevista y otros.

\section{AGRADECIMIENTOS}

Agradecimiento al Ing. Nelson Mollo por facilitarnos de la información académica para la realización de la presente investigación. 


\section{REFERENCIAS}

Benítez, G. \& Ticona, L. (2006). Relación entre el rendimiento del examen de admisión y el académico, Tacna, 2001-2005. Ciencia \& Desarrollo (10), 15- 18.

Cortés, A. \& Palomar, J. (2008). El proceso de admisión como predictor del rendimiento académico en educación superior. Universitas Psychologica, 7 (1), 199-215.

Chávez, M. \& Mendoza; J. (2006). Rendimiento académico de secundaria y su relación con el primer año de educación universitaria en la UN/JBG de Tacna, en el año. Ciencia \& Desarrollo, (10), 47-50.

Flores, M. (2012). Relación entre las modalidades de ingreso y el rendimiento académico de los estudiantes de estomatología de las cohortes 2008-2009 en una Universidad Privada. Revista Estomatológica Herediana 22(4) 210-215.

Larroucau, T. et al (2015). Efecto de la incorporación del ranking de notas en el proceso de admisión a las universidades chilenas. Pensamiento Educativo. Revista de Investigación Educacional Latinoamericana 52(1), 95-118.

Rodríguez, M. \& Gómez, V.M. (2010) Indicadores al ingreso en la carrera de medicina y su relación con el rendimiento académico. Revista de Educación Superior 39(1) 43-50.

Rodríguez C. \& Padilla, G. (2016). Trayectoria Escolar y Ranking: valoraciones y estrategias institucionales en el nuevo escenario de selección universitaria. Revista Estudios Pedagógicos XLII, (3), 313-326.

Treviño Lozano, E. (2003). Relaciones entre el examen de ingreso y las calificaciones con la deserción y la utilidad de la tutoría para la permanencia en Fime de la UANL. Tesis de Grado, Universidad Autónoma de Nueva León, México.

Vergara G. \& Peredo, H. (2017). Relación del desempeño académico de estudiantes de primer año de universidad en Chile y los instrumentos de selección para su ingreso. Revista Educación 41 (2), 2215-2644. 